\title{
Acute appendicitis is characterized by a uniform and highly selective pattern of inflammatory gene expression
}

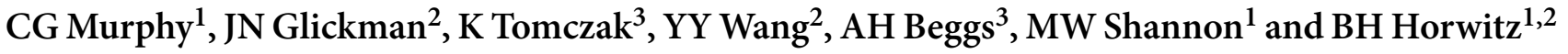

Acute appendicitis (AA) is the most common life-threatening surgical emergency in pediatrics. To characterize the nature of the inflammatory response in AA, gene expression profiles were generated. We found remarkable uniformity in the genes that were differentially expressed between patients with appendicitis and control groups. Sixty-four probe sets were differentially expressed in samples from patients with both severe and mild appendicitis compared to control samples, and within this group we were able to identify four dominant clusters. Interestingly, expression levels of interleukin (IL)-8 significantly correlated with histologic score, and expression of IL-8 protein was observed within both neutrophils and mononuclear cells by immunohistochemistry, suggesting a possible role in the etiology of appendicitis. Although there was some overlap between genes reported to be differentially expressed in Crohn's disease (CD) and those observed in AA, differential expression of genes involved in interferon responses that characterize CD was not observed.

\section{INTRODUCTION}

Acute appendicitis (AA) is a suppurative inflammatory process of the vermiform appendix and is the most common life-threatening surgical emergency in the pediatric age group. ${ }^{1,2}$ Although it is commonly held that obstruction of the appendiceal lumen and subsequent tissue hypoxia and bacterial overgrowth are the precipitating factors of this disease, ${ }^{3,4}$ there is scant evidence to support or refute this hypothesis. Although obstruction of the intestinal lumen by a fecalith is a common finding in AA, large studies suggest that a fecalith is not present in as many as $50 \%$ of cases. ${ }^{5}$ Other mechanisms of functional obstruction such as lymph node hyperplasia have been proposed, but there is little evidence to support this notion, also it is not clear whether AA represents a single disease process or the final common pathway of divergent processes. ${ }^{6}$ Thus, a clear understanding of the pathological basis of appendicitis requires further investigation.

Acute inflammation characterized by infiltration of neutrophils into the mucosa and submucosa is a hallmark of AA. ${ }^{4}$ In addition, alteration in the density of other immune cell populations has been characterized. ${ }^{4}$ Several studies have compared the expression of individual genes in normal and inflamed appendix. Most notably, increased expression of the mRNA for the neutrophil chemoattractant interleukin (IL)-8 has been detected within the mucosa of the inflamed appendix. ${ }^{7}$ Increased amounts of IL-8 protein have also been observed within peritoneal fluid of patients with AA compared to patients without appendicitis, ${ }^{8,9}$ although differences in IL-8 protein levels within the serum have been observed in some but not all studies. ${ }^{8-11}$ Thus, elevated levels of IL-8 are strongly associated with AA.

Interleukin- 8 is generally expressed as part of a wider transcriptional response to inflammatory stimuli such as bacterial lipopolysaccharide or tumor necrosis factor (TNF) and during cell-mediated immune responses. In the digestive tract, elevated expression of IL- 8 within the intestinal mucosa has been observed in patients with both Crohn's disease (CD) and ulcerative colitis (UC). ${ }^{7,12,13}$ The inflammatory response in $\mathrm{CD}$ is associated with a type 1 cell-mediated response including expression of IL-12, interferon (IFN)- $\gamma$, and TNF, ${ }^{14-18}$ whereas the inflammatory response in UC is associated with an atypical type 2 response characterized by expression of IL-5, but not IL-4. ${ }^{17,19}$ Several cell types within the inflamed mucosa have been implicated in the expression of IL-8, including epithelial cells, macrophages, dendritic cells, and neutrophils themselves. ${ }^{13}$ The observations that expression of IL- 8 can be associated with

${ }^{1}$ Division of Emergency Medicine, Children's Hospital, Boston, Massachusetts, USA. ²Department of Pathology, Brigham and Women's Hospital, Boston, Massachusetts, USA. 3Program in Genomics and Division of Genetics, Children's Hospital, Boston, Massachusetts, USA. Correspondence: BH Horwitz (bhorwitz@rics.bwh.harvard.edu) 
Table 1 Characteristics of patients with suspected acute appendicitis and controls

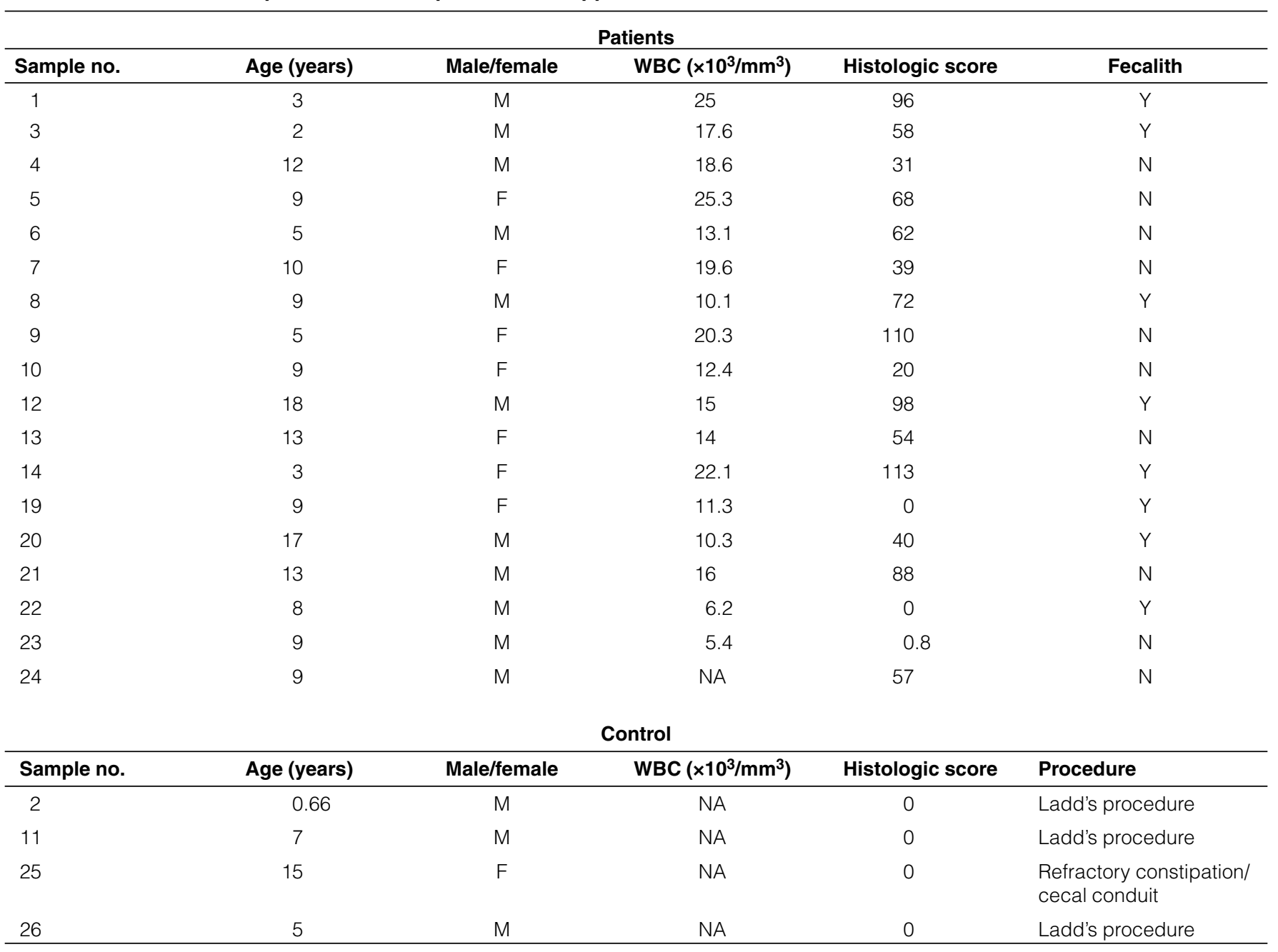

NA, not applicable; WBC, white blood cell.

multiple inflammatory pathways as well as several different cells of origin emphasize the point that understanding the pathways that are activated in conjunction with IL-8 expression may lead to important clues regarding the pathogenesis of AA. ${ }^{13}$

To gain a more comprehensive assessment of the inflammatory response that characterized AA, we have profiled gene expression within the appendices of pediatric patients who were operated on for the diagnosis of suspected AA. Using a newly developed scoring system for severity of appendicitis, we have found a strong correlation between the expression of IL- 8 and the severity of inflammation within the appendix. Furthermore, we have identified a set of 64 probe sets that is highly overexpressed in appendices with both mild and severe inflammation, and found that the genes corresponding to these probe sets can be organized into functional clusters. Interestingly, contrary to our expectations of a broadly based inflammatory process in AA, we did not find evidence for alterations in the expression of many genes characterizing other inflammatory processes within the bowel including IL-12, IFN- $\gamma$, TNF, or IL-5. These results suggest that AA is the result of an intense but tightly controlled innate inflammatory process.

\section{RESULTS}

\section{Correlation of appendicitis score with WBC and IL-8}

All appendices were assigned an appendicitis score based on the criteria described in Methods (Table 1). All specimens obtained from control patients had a histologic score of 0 consistent with the absence of acute inflammation on the histologic level. Specimens obtained from patients who went to the operating room for suspected appendicitis were divided into one of three groups based on appendicitis score of 0 (negative), 1-50 (mild disease), and $>50$ (severe disease). Negative samples represent specimens that were resected for suspicions of appendicitis but which were in fact found to be normal on histologic study. For the purposes of this paper, these specimens are referred to as "negative" samples to emphasize their difference from control specimens. Supplementary Figure S1 online illustrates representative hematoxylin and eosin-stained histological sections of control, mild, and severe samples.

To determine whether there was a relationship between the appendicitis score and other known markers of appendicitis, we correlated the appendicitis score with the peripheral white blood cell (WBC) count and with the expression of IL-8 mRNA within 

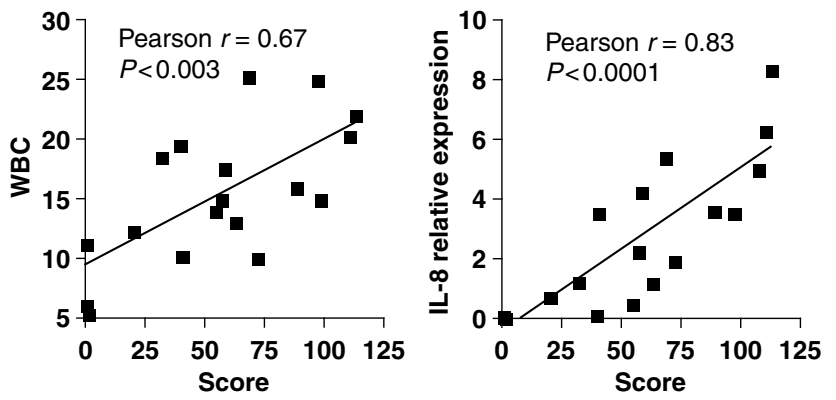

Figure 1 Correlation of histological score with WBC count and IL-8 expression. Graph of histological score and either WBC count (left) or relative expression of IL-8 determined by RPA (right). Line indicates best fit. Pearson $r$ and $P$-value as shown. IL-8, interleukin-8; WBC, white blood cell.

the appendix measured by RNase protection analysis (RPA). There was a significant correlation between both the WBC count and IL-8 expression and the assigned appendix score (Figure 1). Thus, the appendicitis score correlates with known markers of appendicitis and validates the use of this score to gauge severity of disease.

\section{Gene expression in severe appendicitis}

We next selected nine appendix samples for further analysis based on a range of appendicitis scores and IL-8 expression. RNA isolated from these samples as well as that isolated from the four samples removed for noninflammatory reasons (control) was analyzed on Affymetrix U133A 2.0 expression arrays. In the primary analysis, 15,505 of the 22,215 probes corresponding to human genes present on the chips were selected for further analysis based on a flag of present in at least four of the 13 samples. Hierarchical clustering of the samples based on these 15,505 genes revealed two main clusters (Figure 2); one cluster consisting of all the control and negative samples, and a second cluster containing all the mild and severe samples. Thus it appears that the presence or absence of inflammation in the appendix is a dominant feature in the gene expression profiles of these specimens.

To identify probe sets that are differentially expressed in the severe group of samples compared to the control group, we filtered the data for probe sets that had both a fold change of $>5$ or $<-5$ and an uncorrected two-tailed unequal $t$ test threshold of $P<0.05$ between the severe and control groups (Figure 3, top). In all, 276 of the 15,505 probe sets in the analysis group met these criteria (Supplementary Table S1 online). In total, 211 probe sets were upregulated in the severe group, and the 20 probe sets with the largest positive fold change are shown in Table 2. Consistent with the RPA data, two probe sets for IL-8 were included in this list as well as a number of other genes associated with the inflammatory response, including IL-1 $\beta$ and colony stimulating factor-3 (G-CSF). Sixty-five of the 276 selected probe sets were downregulated in the severe group (Supplementary Table S1 online), and the 10 with the largest negative fold change are shown in Table 3. The functional significance of this group is not so clear, but several genes represented by these probe sets are involved in metabolic processes.

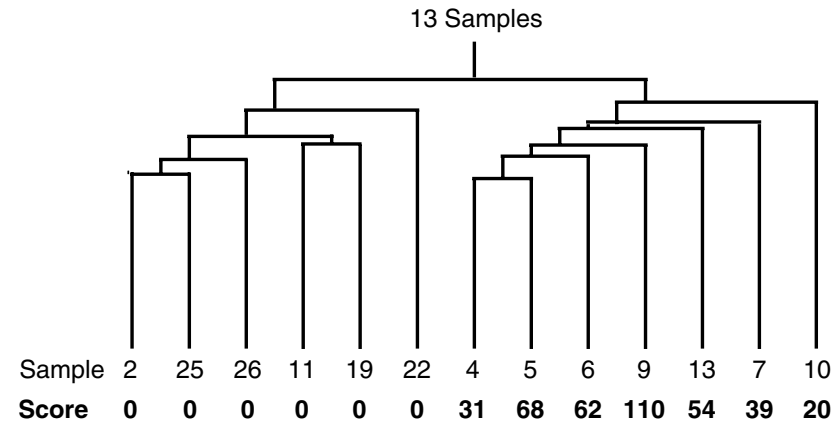

Figure 2 Hierarchical clustering of samples. Microarray data sets were clustered by sample, and the hierarchical tree is shown. Sample number and histology score are shown. Appendices 2, 25, 26, and 11 are controls removed for noninflammatory reasons. Samples 19, 22, 4, 5, 6 , $9,13,7$, and 10 were removed for the preoperative diagnosis of acute appendicitis.

To determine whether probe sets identified in the primary analysis would be identified as differentially expressed by an alternative approach, in a secondary analysis, CEL files were normalized by Robust Multi-Array Analysis, and LIMMA was employed to select probe sets that had a fold change $>5$ or $<-5$ and $P$-value of $<0.05$ using the Benjamini-Hochberg correction for multiple comparisons. ${ }^{20}$ This secondary analysis identified 160 probe sets as differentially expressed between the severe and control groups. Remarkably, 142 of these probe sets were also identified in the primary analysis, and these generally corresponded to those probe sets with the largest fold-change values (Supplementary Table S1 online, italics). Thus, although this secondary analysis may be somewhat more conservative in identifying differentially expressed genes, there is a core set of differentially expressed genes that are common to both methods.

\section{Gene expression in appendix samples with mild inflammation}

To further evaluate the expression of the 276 probe sets that were differentially expressed in the severe samples, expression of these probe sets in all 13 samples was subjected to hierarchical clustering (Figure 3, bottom). These data are displayed as a heat plot with clustered genes on the $y$ axis and samples displayed in the order of lowest to highest histological score, with all four controls furthest to the left. This analysis revealed that there are two predominant clusters corresponding to probe sets that are upregulated and downregulated in the severe group, respectively. Remarkably, there was very similar overall expression of these probes in the mild group compared to samples in the severe group. To better understand the relationship between genes differentially expressed in the mild and severe group, the same filter of both fold change $>5$ or $<-5$, and $P<0.05$, which had been applied to identify genes differentially expressed in the severe group, was applied to a comparison of the mild group with control group. Seventy probe sets met both criteria. Interestingly, of these 70 probe sets, 64 were found in the set of 276 probes that were differentially expressed between the severe and control groups (Supplementary Table S2 online). Fifty-four of these 

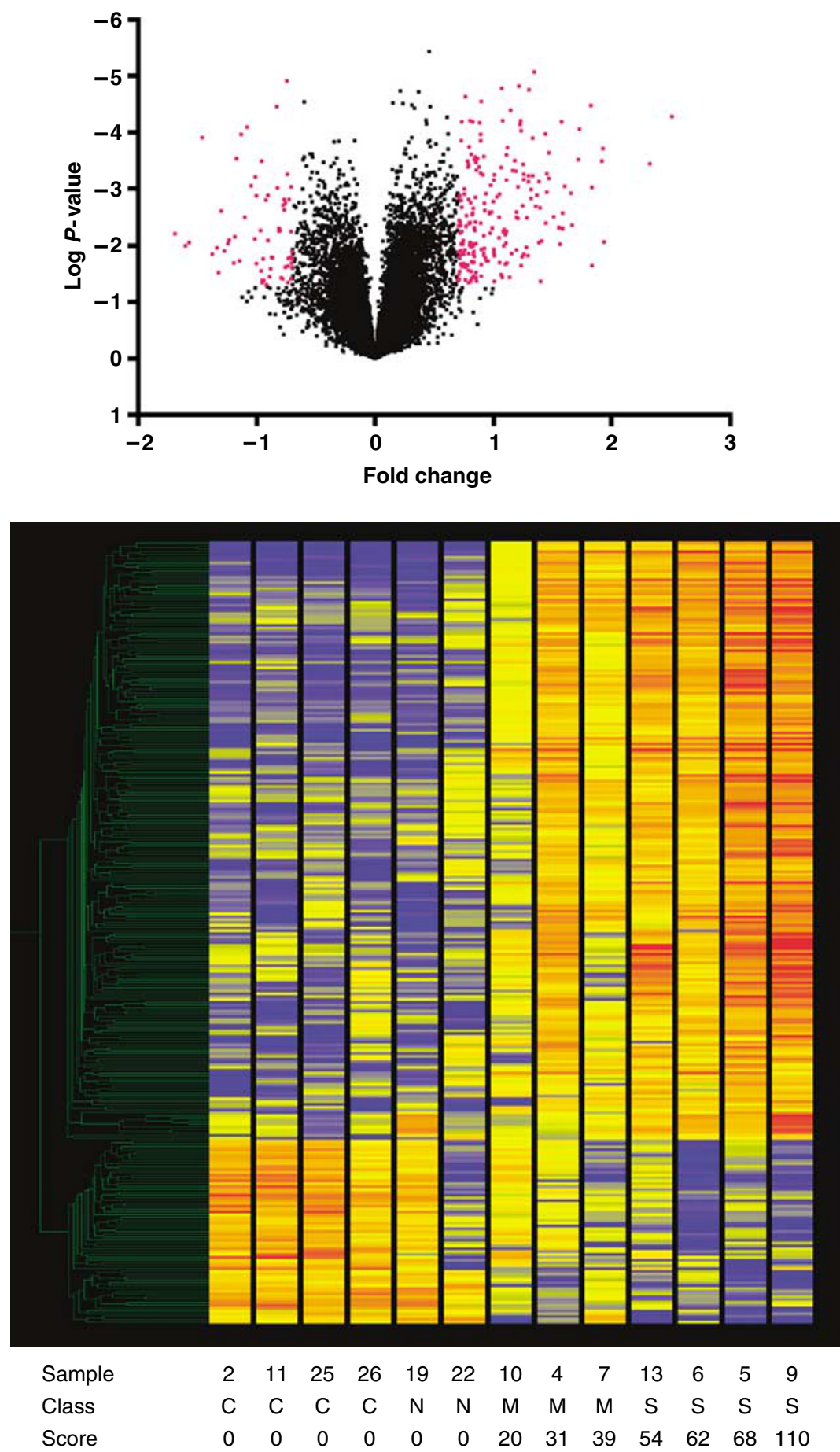

Figure 3 Differential gene expression in severe appendicitis. Top: volcano plot of log $P$-value vs. log fold change comparing the four severe cases of appendicitis with the four control samples. Individual genes shown in pink represent those that have a fold change $>5$ or $<0.2$, and a $P$-value $<0.05$. Bottom: heat map showing hierarchical clustering of the 276 probe sets shown in pink above for all of the 13 appendix samples that were analyzed by microarray. Samples are arranged from lowest to highest histological score, with the four controls on the left.

were upregulated and 10 were downregulated. Thus, there are few genes differentially expressed in the mild group that are not differentially expressed in the severe group, suggesting that cases in the mild group represent either an earlier stage of the inflammatory processor or a less severe process, but not an alternative or independent process. If this is correct, it would suggest that genes differentially expressed in both the mild and severe group may represent those more likely to be involved in the etiology of the pathogenic process, rather than those that are secondarily activated as a result of the inflammatory process.

\section{Functional clustering of differentially expressed genes}

To further explore the function of genes that were differentially expressed in both the mild and severe group, these 64 probe sets 
Table 2 Twenty probe sets with greatest positive fold change in the severe group compared to the control group

\begin{tabular}{|c|c|c|c|}
\hline 206881_s_at & Leukocyte immunoglobulin-like receptor, subfamily A (without TM domain), member 3 & 209.4 & 0.000364 \\
\hline 205220_at & Putative chemokine receptor & 84.67 & 0.000197 \\
\hline 206924_at & Interleukin 11 & 83.22 & 0.000323 \\
\hline 214370_at & S100 calcium binding protein A8 & 67.3 & 0.0229 \\
\hline 205568_at & Aquaporin 9 & 65.89 & $3.35 E-05$ \\
\hline 205936_s_at & Hexokinase 3 (white cell) & 52.63 & $8.73 E-05$ \\
\hline 205863_at & S100 calcium binding protein A12 & 51.65 & 0.000303 \\
\hline 216782_at & Homo sapiens cDNA: FLJ23026 fis, clone LNG01738 & 46.05 & 0.00446 \\
\hline 206522_at & Maltase-glucoamylase (alpha-glucosidase) & 38.24 & 0.00271 \\
\hline 207094_at & Interleukin 8 receptor, alpha & 37.44 & $6.53 E-05$ \\
\hline 208470_s_at & Haptoglobin-related protein & 36.4 & 0.00944 \\
\hline 207442_at & Colony stimulating factor 3 (granulocyte) & 35.62 & 0.00485 \\
\hline 208607_s_at & Serum amyloid A2 & 32.18 & 0.00316 \\
\hline 204007_at & Fc fragment of IgG, low affinity IIIa, receptor for (CD16) & 29.33 & 0.00066 \\
\hline
\end{tabular}

Table 3 Ten probe sets with largest negative fold change in the severe group compared to the control group

\begin{tabular}{llll}
\hline AFFY ID & Description & Fold change & P-value \\
\hline 206149_at & Hepatocellular carcinoma antigen gene 520 & -49.02 & 0.0061 \\
207245_at & UDP glycosyltransferase 2 family, polypeptide B17 & -39.84 & -37.31 \\
220724_at & Hypothetical protein FLJ21511 & -28.82 & 0.0101 \\
220423_at & Phospholipase A2, group IID & -23.75 & 0.0088 \\
206143_at & Solute carrier family 26, member 3 & -21.69 & 0.0143 \\
220834_at & Membrane-spanning 4-domains, subfamily A, member 12 & -20.88 & 0.0109 \\
204130_at & Hydroxysteroid (11-beta) dehydrogenase 2 & -19.96 & 0.0303 \\
220645_at & Chromosome 11 open reading frame 33 & -18.80 & 0.0025 \\
206784_at & Aquaporin 8 & -17.48 & 0.0126 \\
206262_at & Alcohol dehydrogenase 1C (class I), gamma polypeptide & & 0.0093 \\
\hline
\end{tabular}

were functionally clustered using the DAVID Bioinformatics Database Gene Functional Classification Tool. ${ }^{21,22}$ The probe sets corresponded to 54 DAVID IDs and, using the low classification stringency filter, were grouped into four clusters, as well as a group that could not be clustered (Table 4). Cluster 1 contained 20 members and an enrichment score of 6.56. Nineteen of the genes in this cluster were upregulated and one was downregulated compared to control samples. The most significantly enriched GOTERMs within the cluster were receptor, defense response, and immune response, annotations used in 16, 15, and 14 of the 20 genes in this cluster, respectively. This cluster includes the macrophage receptor with collagenous structure, the IL-8 receptor $\alpha$, the colony stimulating factor-3 (G-CSF) receptor, as well as a number of leukocyte immunoglobulin-like receptors. As these receptors are generally expressed on myeloid cells ${ }^{23,24}$ and involved in regulating the recruitment or activation of myeloid cells in inflammatory responses, it is likely that most genes in this cluster are expressed on the surface of macrophages and neutrophils that are responding to an inflammatory stimulus in the appendix. ${ }^{25}$

Cluster 2 has an enrichment score of 4.62 and includes three related S100 calcium-binding proteins (A8, A9, and A12) that are 


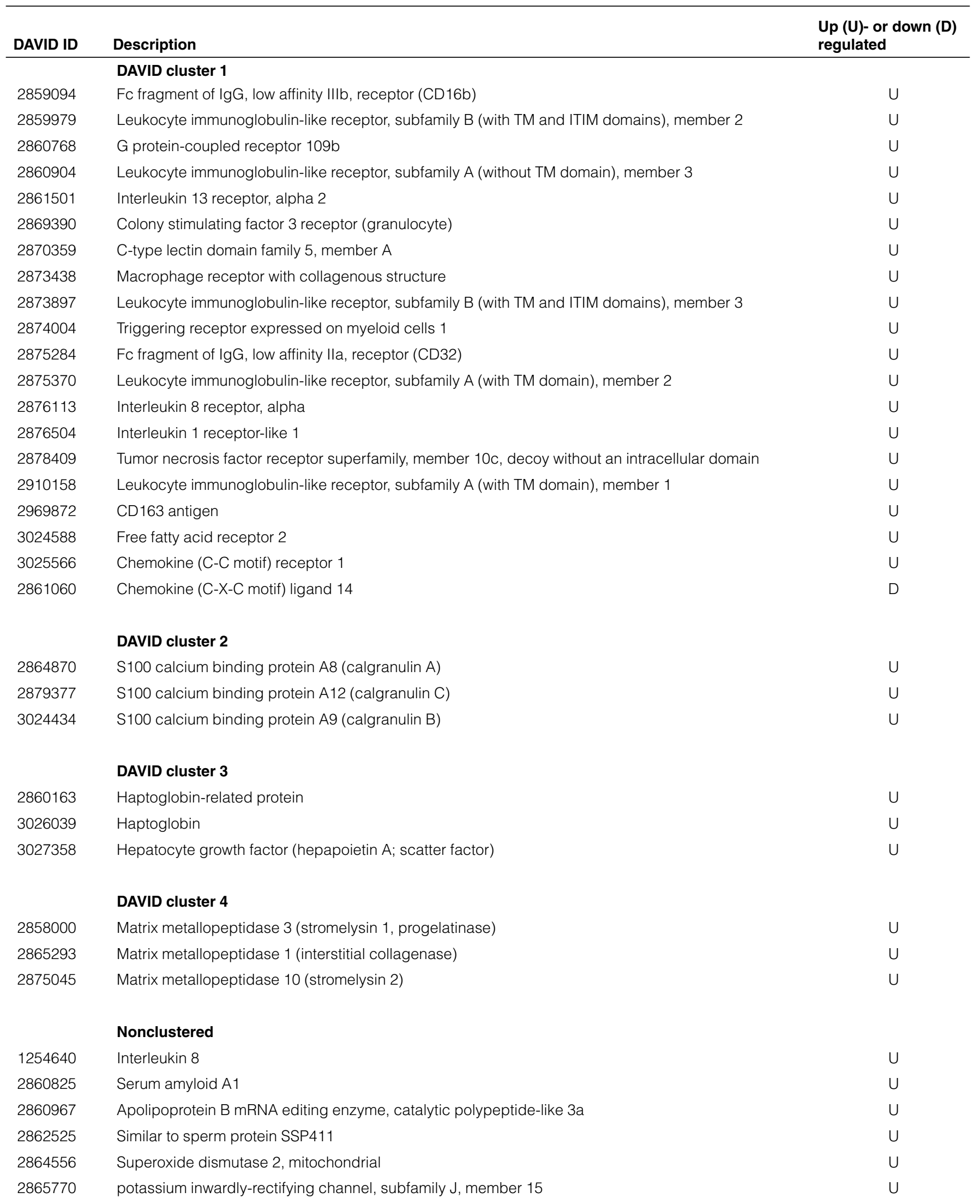




\begin{tabular}{|c|c|c|}
\hline DAVID ID & Description & $\begin{array}{l}\text { Up }(U)-\text { or down }(D) \\
\text { regulated }\end{array}$ \\
\hline 2869048 & Heat shock 70kda protein 6 (HSP70b) & $U$ \\
\hline 2870688 & TIMP metallopeptidase inhibitor 1 & $U$ \\
\hline 2871050 & Interleukin 8 & $U$ \\
\hline 2871098 & Hexokinase 3 (white cell) & $U$ \\
\hline 2872260 & Tissue factor pathway inhibitor 2 & $U$ \\
\hline 2873377 & Serum amyloid $\mathrm{A} 2$ & $U$ \\
\hline 2875756 & Aquaporin 9 & $U$ \\
\hline 2878298 & Interleukin 1 receptor antagonist & $U$ \\
\hline 2878566 & Regenerating islet-derived 1 beta (pancreatic stone protein, pancreatic thread protein) & $U$ \\
\hline 2878817 & Leukocyte immunoglobulin-like receptor, subfamily B (with TM and ITIM domains), member 7 & $U$ \\
\hline 3023714 & Nuclear factor (erythroid-derived 2), 45kda & $U$ \\
\hline 2860141 & Frizzled-related protein & $\mathrm{D}$ \\
\hline 2863181 & ATPase, na+/k+ transporting, alpha $2(+)$ polypeptide & $\mathrm{D}$ \\
\hline 2863569 & Phosphoenolpyruvate carboxykinase 1 (soluble) & $\mathrm{D}$ \\
\hline 2865354 & Butyrylcholinesterase & $\mathrm{D}$ \\
\hline 2870418 & Selenium binding protein 1 & $\mathrm{D}$ \\
\hline 2873008 & 3-hydroxy-3-methylglutaryl-coenzyme a synthase 2 (mitochondrial) & $\mathrm{D}$ \\
\hline 2873479 & Alcohol dehydrogenase $1 \mathrm{~A}$ (class I), alpha polypeptide & $\mathrm{D}$ \\
\hline 3028966 & UDP glucuronosyltransferase 2 family, polypeptide A3 & $\mathrm{D}$ \\
\hline
\end{tabular}

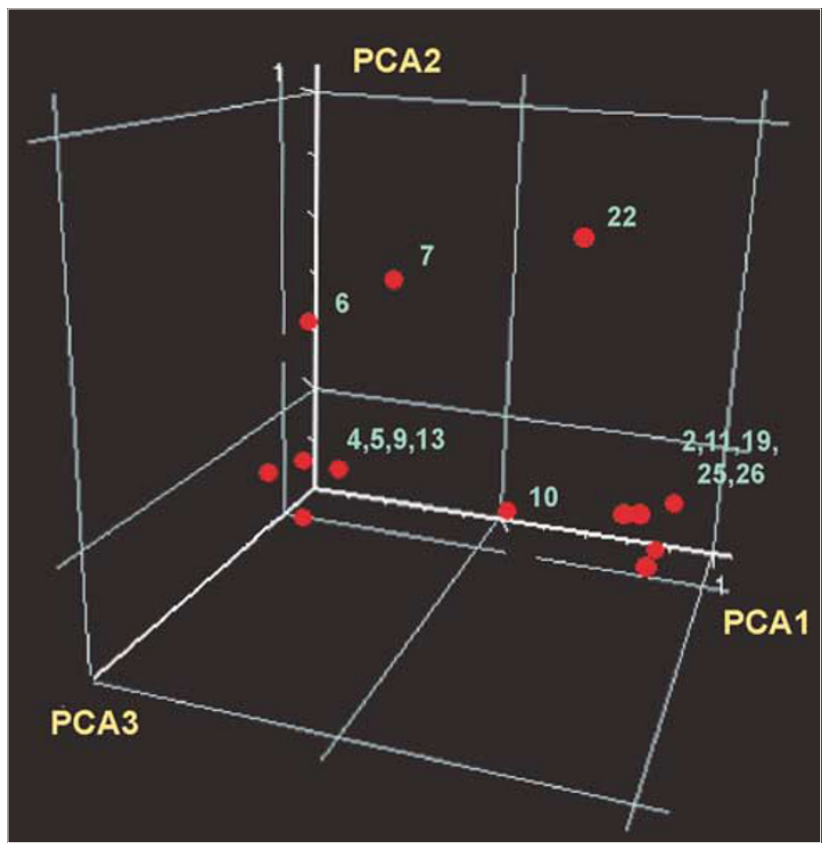

Figure 4 Gene expression in the "negative appendix". Principal component analysis of 276 genes differentially expressed between severe and control groups. Each red dot represents one sample plotted for PCA1, PCA2, and PCA3, as indicated on each axis. Numbers next to individual red dot or cluster of dots indicate corresponding appendix sample(s).

secreted by neutrophils and monocytes. ${ }^{26}$ All have been shown to bind to activated endothelium and may play a role in regulating the adhesion of inflammatory cells to vessel walls. ${ }^{26}$ S100
A12 has also been shown to bind to the receptor for advanced glycation end products on endothelial cells and leukocytes and activate nuclear factor- $\kappa \mathrm{B}$ signaling pathways. ${ }^{27}$ These proteins have previously been used as markers of inflammation in several other inflammatory conditions including cystic fibrosis and Kawasaki's disease. ${ }^{26,28,29}$ Cluster 3 contains hepatocyte growth factor, haptoglobin-related protein, and haptoglobin. All three contain inactive endopeptidase domains. ${ }^{30-33}$ Haptoglobin and haptoglobin-related protein are serum proteins that bind to hemoglobin released in the blood as a result of hemolysis. ${ }^{34}$ Hepatocyte growth factor and haptoglobin are expressed in hepatocytes, whereas transcripts for all three have been identified in neutrophils. ${ }^{34-36}$ Cluster 4 contains the matrix metallopeptidases (MMPs), MMP10, MMP3, and MMP1, proteins involved in extracellular matrix remodeling and strongly associated with a variety of inflammatory responses, including inflammatory bowel disease. Fibroblasts and possibly epithelial cells are thought to be the primary producers of these MMPs. ${ }^{37-39}$ The group of genes that were not clustered includes serum amyloid $\mathrm{A} 1$ and $\mathrm{A} 2$, and interleukin 1 receptor antagonist, serum proteins associated with systemic inflammatory responses; ${ }^{40,41}$ mitochondrial superoxide dismutase, a protein involved in response to oxidative stress; ${ }^{42}$ metallopeptidase inhibitor 1 , an inhibitor of MMP activity that is often expressed in parallel with MMPs; ${ }^{43}$ and IL-8, the potent neutrophil chemoattractant. With one exception (CXCL14), all genes on this list that were downregulated were also not able to be clustered by the DAVID program. Perhaps most fascinating is the observation that on this list, IL-8 is the only definitive chemokine/cytokine 


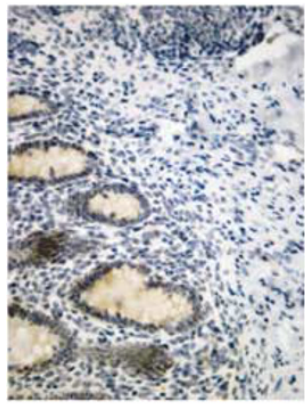

Control

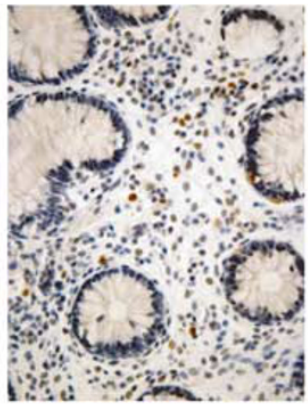

Negative (22)

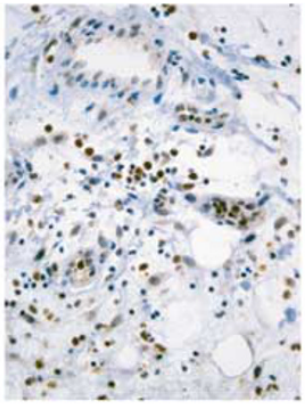

Mild

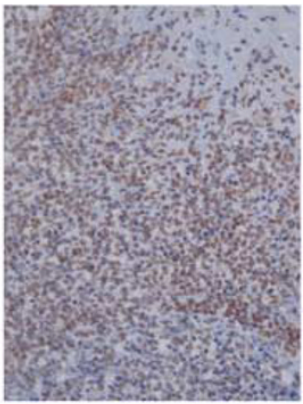

Severe

Figure 5 Immunohistochemical staining for IL-8. Sections of control, negative (no. 22), mild, and severe samples are shown after staining with IL-8 antibody. Original magnification $\times 200$. IL-8, interleukin-8.

that is upregulated. Thus, although several cytokines such as IL-11 and IL-1 are differentially expressed in the severe group, the predominant chemokine/cytokine activity in the mild group is IL-8, suggesting that IL-8 likely plays a central and perhaps unique role in the pathogenesis of appendicitis.

\section{Inflammatory gene expression in the "negative" appendix}

Two appendix samples (nos. 19 and 22) from patients who went to the operating room with the preoperative diagnosis of AA had appendicitis scores of 0 , indicating the absence of histologic signs of inflammation. Both of these patients had symptoms consistent with the diagnosis of appendicitis and a computed tomography scan that showed a fecalith. Fecaliths were found on the pathologic assessment of both appendix samples. Although these appendices showed no histologic signs of AA, we wondered whether either of these two samples might show some of the molecular characteristics of appendicitis that we have characterized above. Visual inspection of the heat map, representing genes differentially expressed in samples of severe appendicitis (Figure 3, bottom), suggested that although sample 19 was very similar to control samples, there appeared to be differential expression of several subclusters of these genes in sample 22 compared to the control samples. Interestingly, this included genes that were both upregulated and downregulated. To better define the relationship between gene expression in sample 22 and expression in both the control group and the group with proven appendicitis, we performed a principal component analysis on the 276 probe sets that were differentially expressed in the severe group (Figure 4). Three principal components were identified. Principal component 1 (PCA1) explained $72.2 \%$ of the variance in the samples, whereas principal components 2 and 3 had less prominent roles in explaining variance ( 7.7 and 3.92\%, respectively). Examining the distribution of samples by values of PCA1, PCA2, and PCA3 revealed that sample 19 was part of a cluster that included all four control samples, suggesting that sample 19 is a true negative. Interestingly, sample 22 exhibited a lower PCA1 value than any sample in the control group and did not cluster with the negative controls. These data raise the possibility that sample 22 could represent a very early stage of inflammation that exists after the development of obstructive physiology but before the detection of overt inflammation. If correct, genes differentially expressed at this stage could hold important clues regarding the pathophysiology of appendicitis. Firm conclusions regarding this possibility will require isolating and analyzing additional samples from patients with fecaliths but without signs of inflammation on histologic evaluation.

\section{Protein expression of IL-8 within the appendix}

To further characterize the expression of IL- 8 during AA, we used an antibody directed at IL- 8 to stain sections of control and inflamed appendix samples (Figure 5). Although there was little specific staining in control samples, sections derived from samples with severe inflammation revealed intense IL-8 staining within infiltrating neutrophils, as well as within mononuclear cells. Interestingly, IL-8-positive mononuclear cells were also observed within sections derived from samples with mild inflammation and sparse neutrophilic infiltration, whereas there was considerably less staining within neutrophils. IL-8positive mononuclear cells were observed predominantly within the interfollicular ( $\mathrm{T}$ cell) zones rather than germinal centers, suggesting that these cells may be activated $\mathrm{T}$ cells, although further analysis will be necessary to definitively establish the mononuclear cell type expressing IL-8. Remarkably, IL-8-positive mononuclear cells are also detected within negative sample 22 , despite the complete absence of neutrophils. These results suggest that expression of IL-8 by mononuclear cells precedes neutrophil infiltration, raising the possibility that this represents a very early stage in the pathogenic process.

\section{Comparison of gene expression in acute appendicitis and inflammatory bowel disease}

Elevated expression of IL-8 has been observed in other inflammatory processes within the bowel, including $\mathrm{CD}$ and UC.,12,13 Therefore, we wondered whether there might be similarity between the inflammatory response observed in inflammatory bowel diseases and AA, especially with regard to elevated expression of genes associated with type 1 responses observed in CD. ${ }^{15,17-19} \mathrm{~A}$ recent publication has compared global expression profiles in endoscopic biopsy specimens from patients with $\mathrm{CD}$ and UC to normal controls, using Affymetrix expression arrays. ${ }^{44}$ This study found elevated expression of acute inflammatory genes in both $\mathrm{CD}$ and $\mathrm{UC}$, and elevated expression of genes involved in antigen presentation and the interferon response specifically in $\mathrm{CD} .{ }^{44}$ Although the reported data were 


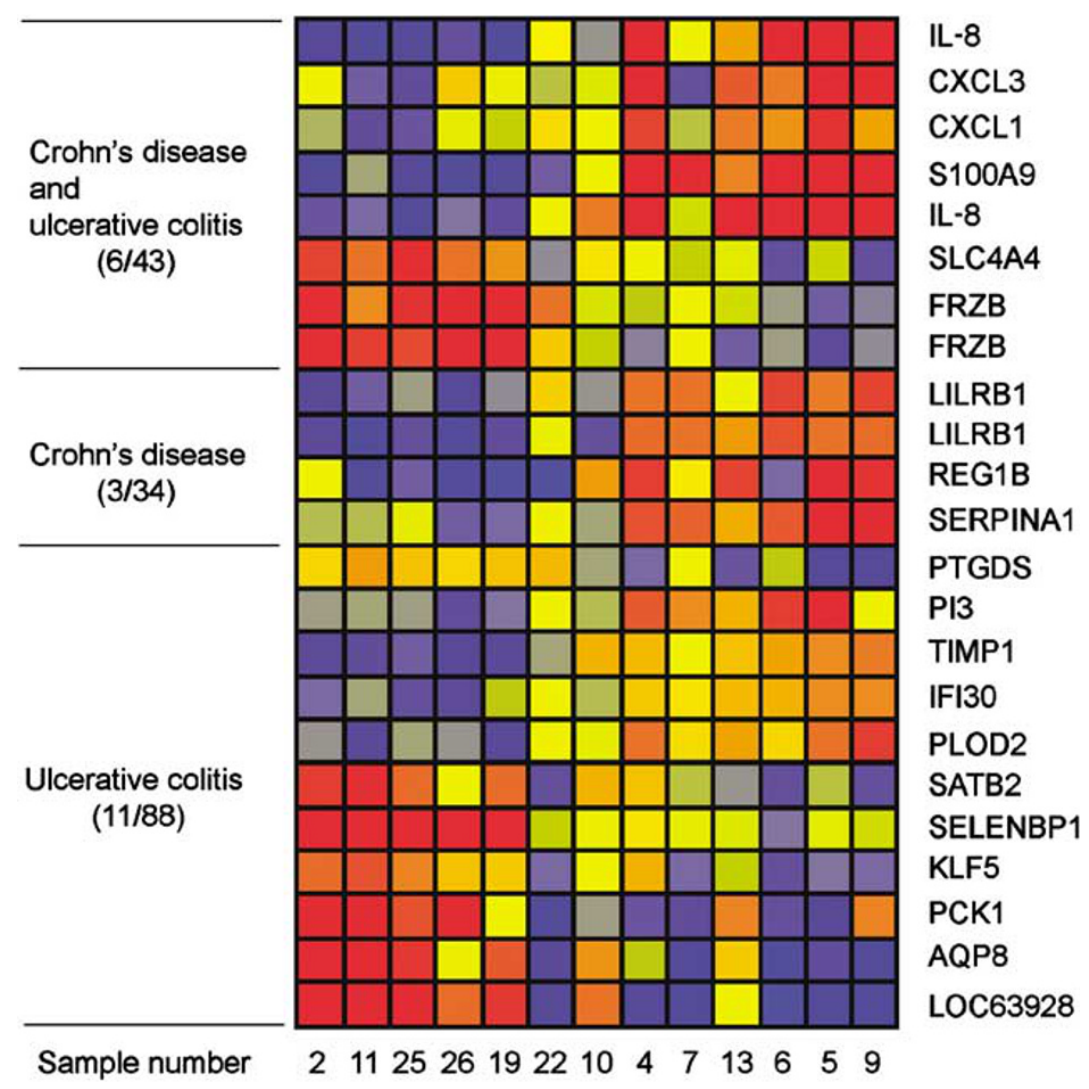

Figure 6 Heat plot of genes differentially expressed in IBD, which are also differentially expressed in AA. Samples are arranged from lowest to highest score with four controls on the left. Genes that are differentially expressed in both AA and IBD are shown. Genes are grouped based on being differentially expressed in either both $C D$ and $U C, C D$ alone, or $U C$ alone, according to ref. 44 . Numbers in parentheses refer to the number of genes differentially expressed in both AA and IBD compared to the total number of genes differentially expressed in IBD for each group. Gene symbols are shown on the right. Please note that several genes (IL-8, FRZB, LILRB1) are included twice in a group because two probe sets corresponding to these gene symbols were differentially expressed in AA. These genes are included only once in the numbers in parentheses of genes differentially expressed in both AA and IBD. AA, acute appendicitis; CD, Crohn's disease; IBD, inflammatory bowel disease; IL-8, interleukin-8; UC, ulcerative colitis.

generated with a different platform (U95Av2) than what we used in the current study, precluding direct comparison, we did search our list of 276 probe sets that were differentially expressed in samples of severe appendicitis for associated gene symbols that were identified in the previous study as differentially expressed in either both CD and UC, CD alone, or UC alone (see Figure 4 and Tables 4-6 in ref. 44). We found that probe sets corresponding to 6 of 43 gene symbols differentially expressed in both CD and UC, 3 of 34 differentially expressed in $\mathrm{CD}$ alone, and 11 of 88 differentially expressed in UC alone were also differentially expressed in severe appendicitis, and altered expression of many of these genes could also be observed in the cases of more mild appendicitis (Figure 6). The direction of differential expression (up- or downregulated) in AA paralleled the direction observed in inflammatory bowel disease (IBD) for all but one gene (prostaglandin D2 synthase (PTGDS)). Genes common to AA and IBD included IL-8 as well as several genes presumably involved in generation of the acute inflammatory response such as CXCL1, CXCL3, and S100 binding protein A9 (S100A9). Interestingly, a number of genes were also downregulated in both AA and UC, including aquaporin 8 (AQP8) and phosphoenolpyruvate carboxykinase 1 (PCK1). However, none of the genes involved in antigen presentation or interferon response that were differentially expressed in $\mathrm{CD}^{44}$ were among the genes that we identified as differentially expressed in AA. Furthermore, we performed independent analysis of AA samples using RPA and verified that the expression of TNF, IL-12 p40, IFN- $\gamma$, IL-4, IL-2, and IL-15 was quite low and showed no clear change between control and inflamed samples (data not shown). Thus, as expected, although there is some overlap between the expression of acute inflammatory genes in AA and IBD, there is no evidence for the activation of a type 1 or interferon-mediated response in AA, suggesting that it is mediated by a distinct pathophysiologic process.

\section{DISCUSSION}

Although it is well recognized that appendices removed for the diagnosis of appendicitis can visually display a wide range of morphologic inflammatory changes, to our knowledge there have been few attempts to quantify the severity of inflammation and to correlate morphologic features with gene expression. Here we have developed a scoring system and demonstrated a significant correlation between the score and both WBC count and IL-8 levels, known markers of appendicitis, suggesting that 
the appendicitis score is a valid measure of inflammation severity for the purposes of our study. Scoring samples then allowed us to stratify samples into absent, mild, or severe inflammation. Interestingly, however, the gene expression profiles of both the severe and mild group appeared very similar with the vast majority of genes that were differentially expressed in the mild group also differentially expressed in the severe group. Although this analysis does not rule out the possibility that there are biologically important differences in the nature of the inflammatory response in different samples, it does demonstrate that a core set of inflammatory genes are activated in all samples regardless of the degree of inflammation, suggesting there is a uniform inflammatory response underlying the pathological basis of appendicitis. One caveat to this interpretation is that none of the samples in either the severe or mild group that underwent microarray analysis were noted to have fecaliths present on pathological examination, and thus it is possible that there are variations in the inflammatory response in samples with a fecalith. However, using RPA, we did evaluate expression of several inflammatory genes including IL-8 in appendix samples that were noted to have fecaliths, and found little difference in the nature of inflammatory gene expression for samples with and without fecaliths (Figure 1, and data not shown). Nonetheless, the issue of whether there are differences in the global inflammatory response in samples with and without the presence of a fecalith requires further study.

Many chronic inflammatory responses in the bowel have been found to show either a Th1 or a Th2 lymphocyte bias. ${ }^{45,46}$ Although AA is thought to be an acute process, the fact that it occurs in a highly active immune environment raised the possibility that AA could be the end result of an ongoing T cellmediated response. Furthermore, it has been suggested that AA may be associated with some forms of IBD. ${ }^{47,48}$ However, we found no evidence of a Th1 or Th2 response in the inflammatory process of AA. One caveat to the list of genes that were identified using microarray analysis is that because of the robust dysregulation of acute inflammatory genes, subtle alteration in potentially important regulatory genes could be difficult to appreciate. Nonetheless, direct assessment of several critical regulatory genes such as IL-12 p40, IFN- $\gamma$, and IL-4 using RPA did not reveal significant differences (data not shown). Furthermore, we found no evidence for a Th17 response in appendicitis, as expression of both IL-17a and IL-23 p19 mRNA was at the lower limits of detection using quantitative real-time PCR, whereas as expected we were easily able to detect robust expression of IL-8 mRNA within inflamed samples using the same technique (data not shown). Nonetheless, our finding of IL-8 expression by mononuclear cells does raise the possibility that some other type of lymphocyte-mediated response is involved in the pathogenesis of appendicitis. This possibility will be evaluated in future studies.

Perhaps the most remarkable finding in this study is the highly selective induction of mediators of the innate inflammatory response. IL-1 and TNF are both strongly induced by bacterial products such as lipopolysaccharide and are themselves potent activators of the inflammatory response. However, although we found evidence for potent induction of IL- $1 \beta$ in samples of AA, there was no detectable induction of TNF. This indicates that despite the impressive histological appearance of AA, the underlying inflammatory response is a highly focused process, suggesting the activation of highly specific inflammatory pathways. Identifying these pathways is clearly a highly relevant topic for additional investigation.

Appendicitis is characterized by the infiltration of neutrophils into the appendix, ${ }^{4}$ and we have used the intensity of this infiltration as the primary factor in assigning appendicitis scores in this study. Thus, a reasonable question is whether the alterations in gene expression that we have observed are the result of changes in the gene expression profiles of resident cells within the appendix or alternatively reflect the genes being expressed by these infiltrating neutrophils. Using immunohistochemistry, we have found that mononuclear cells rather than neutrophils are the predominant cell type expressing IL-8 in samples with mild inflammation. Although further analysis will be necessary to definitively establish the identity of the mononuclear cell type expressing IL-8, this analysis suggests that there are alterations of inflammatory gene expression within the appendix prior to the infiltration of neutrophils.

In conclusion, this study demonstrates that AA is characterized by a reproducible pattern of inflammatory gene expression suggesting a uniform underlying pathogenesis. The relatively circumscribed pattern of observed inflammatory gene expression indicates that the inflammatory stimulus driving disease is acting with a high degree of specificity, and is unlikely to be related to type 1 or 2 immune responses that characterize other inflammatory diseases of the lower bowel. We suggest that a detailed understanding of events that occur in the earliest stages of inflammation could lead to important clues regarding disease pathogenesis, and that understanding the pathogenesis of this highly prevalent disease could ultimately lead to more accurate early diagnosis, and influence strategies for intervention.

\section{METHODS}

Selection of patients and controls. A convenience sample of 22 appendix specimens was obtained from patients whose appendices were resected at a tertiary care children's hospital over a 2-year period. Eighteen of these 22 specimens were obtained from patients who went to the operating room with the preoperative diagnosis of suspected AA (Table 1). The remaining four samples were obtained from individuals who were undergoing intra-abdominal surgery for a noninflammatory condition and whose appendix was electively resected during this procedure (Table 1). These latter four specimens will be referred to as controls.

Appendicitis score. All hematoxylin and eosin-stained slides from each case were reviewed by one of the authors (J.G.) without knowledge of clinical data or experimental results. A histologic grading score to estimate the severity of the appendicitis was generated for each sample. This appendicitis score was the product of the maximum number of neutrophils per high-power field and the percent of each tissue section that was infiltrated by neutrophils. The appendicitis score ranges from 0 to 113.

RNase protection analysis. A circumferential piece of tissue was obtained at the distal aspect of each specimen. The tissue was flash frozen at $-80^{\circ} \mathrm{C}$ for later processing. Frozen specimens were 
homogenized into TriReagent (Molecular Research Center, Cincinnati, $\mathrm{OH}$ ) and RNA was isolated according to the manufacturer's instruction. RPAs were performed on 10-20 $\mu$ g of total RNA using RiboQuant Multi Probe Template Sets (BD PharMingen, San Diego, CA). Intensities of the protected fragments corresponding to IL- 8 were quantified by phosphorimager analysis and expressed as a ratio, relative to GAPDH, which was included as an internal control for each sample.

Correlation analysis. Correlation analysis between WBC count and appendicitis score, as well as relative IL-8 expression and appendicitis score was performed using GraphPad Prism 4 software (GraphPad Software, San Diego, CA).

Gene array and data analysis. Targets were prepared from RNA isolated as above and hybridized to Affymetrix U133A 2.0 Array as previously described. ${ }^{49}$ U133A 2.0 Arrays contain 18,400 transcripts and variants, including 14,500 well-characterized human genes. In the primary analysis, the 13 data sets were first processed by Affymetrix Gene Chip Operating System (GCOS) V.1.4.0.036 software, which computed present/absent calls for each probe set, and the signal values were adjusted to an overall target intensity of 1500 . Data were then imported into Genespring software Version 7.3.1 and normalized in three steps: (1) data transformation: set measurements less than 0.01 to 0.01 ; (2) per chip: normalization to 50th percentile; (3) per gene: normalize to median. Hierarchical clustering by both samples and genes, and principal component analysis were performed using Genespring. Probe sets were functionally clustered using the DAVID Bioinformatics Database Gene Functional Classification Tool. ${ }^{21,22}$

In a second analysis, expression data were read from CEL files and background correction, normalization, and summarization of the probe-level data into probe set expression values were accomplished using Robust Multi-Array Analysis, and differential expression analyzed using the LIMMA package of the afflylmGUI within the Bioconductor R environment. ${ }^{20}$ The microarray data discussed in this publication have been deposited in NCBIs Gene Expression Omnibus (GEO, http://www. ncbi.nlm.nih.gov/geo/) and are accessible through GEO Series accession number GSE9579.

Immunohistochemistry. Immunohistochemical staining was performed using an IL-8 rabbit polyclonal antibody (Santa Cruz Biotechnology, Santa Cruz, CA).

Institutional review. The study was approved by the Institutional Review Board at Children's Hospital, Boston, and all data collection was compliant with the Health Insurance Portability and Accountability Act of 1996.

SUPPLEMENTARY MATERIAL is linked to the online version of the paper at http://www.nature.com/mi

\section{ACKNOWLEDGMENTS}

We thank Mei Han for her invaluable assistance in generation of the microarray data sets. Support to BHH from NIH AI52267, and a Crohn's and Colitis Foundation of America and the William and Shelby Modell Family Foundation Senior Research Grant. Support to KT and AHB from NIH, NIAMS AR044345 and the Muscular Dystrophy Association. Microarray data were generated in the Children's Hospital Boston Genomic Microarray Core Laboratory supported by NIH, NINDS NS40828.

\section{DISCLOSURE}

The authors declared no conflict of interest.

(c) 2008 Society for Mucosal Immunology

\section{REFERENCES}

1. Addiss, D.G., Shaffer, N., Fowler, B.S. \& Tauxe, R.V. The epidemiology of appendicitis and appendectomy in the United States. Am. J. Epidemiol. 132, 910-925 (1990).
2. Scholer, S.J., Pituch, K., Orr, D.P. \& Dittus, R.S. Clinical outcomes of children with acute abdominal pain. Pediatrics 98, 680-685 (1996).

3. Puri, P., Rangecroft, L., Zervos, G., Guiney, E.J. \& O'Donnell, B. Perforated appendicitis in children: use of metronidazole for the reduction of septic complications. Z. Kinderchir. 32, 111-115 (1981).

4. Tsuji, M., Puri, P. \& Reen, D.J. Characterisation of the local inflammatory response in appendicitis. J. Pediatr. Gastroenterol. Nutr. 16, 43-48 (1993).

5. Jones, B.A., Demetriades, D., Segal, I. \& Burkitt, D.P. The prevalence of appendiceal fecaliths in patients with and without appendicitis. A comparative study from Canada and South Africa. Ann. Surg. 202, 80-82 (1985).

6. Babekir, A.R. \& Devi, N. Analysis of the pathology of 405 appendices. East. Afr. Med. J. 67, 599-602 (1990).

7. Mazzucchelli, L. et al. Expression of interleukin-8 gene in inflammatory bowel disease is related to the histological grade of active inflammation. Am. J. Pathol. 144, 997-1007 (1994).

8. Dalal, I. et al. Serum and peritoneal inflammatory mediators in children with suspected acute appendicitis. Arch. Surg. 140, 169-173 (2005).

9. Zeillemaker, A.M. et al. Peritoneal interleukin-8 in acute appendicitis. J. Surg. Res. 62, 273-277 (1996).

10. Yoon, D.Y. et al. Human cytokine levels in nonperforated versus perforated appendicitis: molecular serum markers for extent of disease? Am. Surg. 68, 1033-1037 (2002).

11. Paajanen, H. et al. Novel serum inflammatory markers in acute appendicitis. Scand. J. Clin. Lab. Invest. 62, 579-584 (2002).

12. Daig, R. et al. Increased interleukin 8 expression in the colon mucosa of patients with inflammatory bowel disease. Gut 38, 216-222 (1996).

13. Grimm, M.C., Elsbury, S.K., Pavli, P. \& Doe, W.F. Interleukin 8: cells of origin in inflammatory bowel disease. Gut 38, 90-98 (1996).

14. Kosiewicz, M.M. et al. Th1-type responses mediate spontaneous ileitis in a novel murine model of Crohn's disease. J. Clin. Invest. 107, 695-702 (2001).

15. Matsuoka, K. et al. T-bet upregulation and subsequent interleukin 12 stimulation are essential for induction of Th1 mediated immunopathology in Crohn's disease. Gut 53, 1303-1308 (2004).

16. Powrie, F. et al. Inhibition of Th1 responses prevents inflammatory bowel disease in scid mice reconstituted with CD45RBhi CD4. T cells. Immunity 1, 553-562 (1994).

17. Monteleone, I., Vavassori, P., Biancone, L., Monteleone, G. \& Pallone, F. Immunoregulation in the gut: success and failures in human disease. Gut 50 (Suppl 3), III60-III64 (2002).

18. Parronchi, P. et al. Type 1 T-helper cell predominance and interleukin-12 expression in the gut of patients with Crohn's disease. Am. J. Pathol. 150, 823-832 (1997).

19. Fuss, I.J. et al. Disparate CD4 lamina propria (LP) lymphokine secretion profiles in inflammatory bowel disease. Crohn's disease LP cells manifest increased secretion of IFN-gamma, whereas ulcerative colitis LP cells manifest increased secretion of IL-5. J. Immunol. 157, 1261-1270 (1996).

20. Wettenhall, J.M., Simpson, K.M., Satterley, K. \& Smyth, G.K. affylmGUI: a graphical user interface for linear modeling of single channel microarray data. Bioinformatics 22, 897-899 (2006).

21. Dennis, G. Jr et al. DAVID: database for annotation, visualization, and integrated discovery. Genome Biol. 4, P3 (2003).

22. Hosack, D.A., Dennis, G. Jr, Sherman, B.T., Lane, H.C. \& Lempicki, R.A. Identifying biological themes within lists of genes with EASE. Genome Biol. 4, R70 (2003).

23. Bertrand, G. et al. Use of serial analysis of gene expression (SAGE) technology reveals new granulocytic markers. J. Immunol. Methods 292, 43-58 (2004).

24. Theilgaard-Monch, K. et al. The transcriptional program of terminal granulocytic differentiation. Blood 105, 1785-1796 (2005).

25. Kraal, G., van der Laan, L.J., Elomaa, O. \& Tryggvason, K. The macrophage receptor MARCO. Microbes Infect. 2, 313-316 (2000).

26. Roth, J., Vogl, T., Sorg, C. \& Sunderkotter, C. Phagocyte-specific S100 proteins: a novel group of proinflammatory molecules. Trends Immunol. 24, 155-158 (2003).

27. Herold, K. et al. Receptor for advanced glycation end products (RAGE) in a dash to the rescue: inflammatory signals gone awry in the primal response to stress. J. Leukoc. Biol. 82, 204-212 (2007).

28. Heizmann, C.W. The importance of calcium-binding proteins in childhood diseases. J. Pediatr. 147, 731-738 (2005). 
29. Robinson, M.J. \& Hogg, N. A comparison of human S100A12 with MRP-14 (S100A9). Biochem. Biophys. Res. Commun. 275, 865-870 (2000).

30. Nakamura, T. et al. Molecular cloning and expression of human hepatocyte growth factor. Nature 342, 440-443 (1989).

31. Bensi, G., Raugei, G., Klefenz, H. \& Cortese, R. Structure and expression of the human haptoglobin locus. EMBO J. 4, 119-126 (1985)

32. Kurosky, A. et al. Covalent structure of human haptoglobin: a serine protease homolog. Proc. Natl. Acad. Sci. USA 77, 3388-3392 (1980).

33. Maeda, N. Nucleotide sequence of the haptoglobin and haptoglobin-related gene pair. The haptoglobin-related gene contains a retrovirus-like element. J. Biol. Chem. 260, 6698-6709 (1985).

34. Nielsen, M.J. et al. Haptoglobin-related protein is a high-affinity hemoglobin-binding plasma protein. Blood 108, 2846-2849 (2006).

35. Grenier, A. et al. Presence of a mobilizable intracellular pool of hepatocyte growth factor in human polymorphonuclear neutrophils. Blood 99, 2997-3004 (2002).

36. Theilgaard-Monch, K. et al. Haptoglobin is synthesized during granulocyte differentiation, stored in specific granules, and released by neutrophils in response to activation. Blood 108, 353-361 (2006).

37. Gueders, M.M., Foidart, J.M., Noel, A. \& Cataldo, D.D. Matrix metalloproteinases (MMPs) and tissue inhibitors of MMPs in the respiratory tract: potential implications in asthma and other lung diseases. Eur. J. Pharmacol. 533, 133-144 (2006).

38. Medina, C. \& Radomski, M.W. Role of matrix metalloproteinases in intestinal inflammation. J. Pharmacol. Exp. Ther. 318, 933-938 (2006).
39. Goetzl, E.J., Banda, M.J. \& Leppert, D. Matrix metalloproteinases in immunity. J. Immunol. 156, 1-4 (1996).

40. Jensen, L.E. \& Whitehead, A.S. Regulation of serum amyloid A protein expression during the acute-phase response. Biochem. J. 334 (Part 3), 489-503 (1998).

41. Arend, W.P., Malyak, M., Guthridge, C.J. \& Gabay, C. Interleukin-1 receptor antagonist: role in biology. Annu. Rev. Immunol. 16, 27-55 (1998).

42. Robinson, B.H. The role of manganese superoxide dismutase in health and disease. J. Inherit. Metab. Dis. 21, 598-603 (1998).

43. Coussens, L.M. \& Werb, Z. Matrix metalloproteinases and the development of cancer. Chem. Biol. 3, 895-904 (1996).

44. Wu, F. et al. Genome-wide gene expression differences in Crohn's disease and ulcerative colitis from endoscopic pinch biopsies: insights into distinctive pathogenesis. Inflamm. Bowel. Dis. 13, 807-821 (2007).

45. Podolsky, D.K. Inflammatory bowel disease. N. Engl. J. Med. 347, 417-429 (2002).

46. Neurath, M.F., Finotto, S. \& Glimcher, L.H. The role of Th1/Th2 polarization in mucosal immunity. Nat. Med. 8, 567-573 (2002)

47. Koutroubakis, I.E., Vlachonikolis, I.G. \& Kouroumalis, E.A. Role of appendicitis and appendectomy in the pathogenesis of ulcerative colitis: a critical review. Inflamm. Bowel. Dis. 8, 277-286 (2002)

48. Scott, I.S., Sheaff, M., Coumbe, A., Feakins, R.M. \& Rampton, D.S. Appendiceal inflammation in ulcerative colitis. Histopathology 33, 168-173 (1998).

49. Cerletti, M. et al. Melanoma cell adhesion molecule is a novel marker for human fetal myogenic cells and affects myoblast fusion. J. Cell Sci. 119, 3117-3127 (2006). 